\title{
TTR
}

Traduction, terminologie, re?daction

\section{À partir de Georges Mounin : esquisse archéologique}

\section{Jean-René Ladmiral}

Volume 8, numéro 1, 1er semestre 1995

Orientations européennes en traductologie

URI : https://id.erudit.org/iderudit/037196ar

DOI : https://doi.org/10.7202/037196ar

Aller au sommaire du numéro

\section{Éditeur(s)}

Association canadienne de traductologie

\section{ISSN}

0835-8443 (imprimé)

1708-2188 (numérique)

Découvrir la revue

\section{Citer cet article}

Ladmiral, J.-R. (1995). À partir de Georges Mounin : esquisse archéologique. TTR, 8(1), 35-64. https://doi.org/10.7202/037196ar

\section{Résumé de l'article}

À partir de Georges Mounin : esquisse archéologique - Georges Mounin est l'un des « pères fondateurs » de la traductologie: c'est donc à partir de cet auteur qu'on pourra entreprendre une archéologie de la discipline - au double sens où c'est à la fois vouloir le dépasser, " en partir ", et où c'est aussi " partir " de ses travaux. Certes, il faut récuser la problématique de robjection préjudicielle, qui nous confronte aux apories de l'« intraduisible », en réinscrivant dans l'histoire les problèmes de la traduction. Encore devra-t-on ne pas se contenter d'une traductologie purement linguistique, voire d'une " traductologie de la langue ». Plus généralement, dans la foulée de l'héritage de Mounin, on sera amené à poser le problème de l'articulation entre traductologie et linguistique; on en viendra à s'interroger sur le statut du discours traductologique, sur la dimension philosophique qui est la sienne et sur la place qui lui revient dans le champ épistémologique des sciences humaines.
Tous droits réservés ( $\mathrm{TTR}$ : traduction, terminologie, rédaction - Les auteurs, 1995 document est protégé par la loi sur le droit d'auteur. L'utilisation des services d'Érudit (y compris la reproduction) est assujettie à sa politique d'utilisation que vous pouvez consulter en ligne. 


\section{À partir de Georges Mounin: esquisse archéologique}

\section{Jean-René Ladmiral}

\section{Continuité et dépassement}

En écrivant les pages qu'on va lire, mon intention n'est pas tant de rendre un hommage posthume au regretté Georges Mounin, de rédiger en son honneur ce qu'il est convenu d'appeler traditionnellement un «tombeau». Encore moins s'agit-il ici d'un article sur Mounin - qui s'attacherait à faire le bilan de son apport, important, dans le domaine de la traduction. Il convient d'entendre l'archéologie, dont j'annonce ici l'esquisse, au sens «foucaldien» du terme, c'est-à-dire au sens d'un discours qui se situe à l'articulation de l'épistémologie et de l'histoire des sciences, de l'histoire des sciences humaines - et, en l'occurrence, de l'histoire de cette science humaine nouvelle que constitue la traductologie'. Plus spécifiquement, je tiendrai ici le discours d'une «auto-archéologie», réunissant quelques éléments pour une reconstruction rétrospective de la logique de la recherche qui a été la mienne en traductologie; et d'abord il s'agissait esquisser le contexte historique dans la

1. Michel Foucault, les Mots et les choses. Une archéologie des sciences humaines (Paris, Gallimard, 1966). 
mesure où, comme le rappelle justement René Lourau, «il n'y a pas de genèse intellectuelle sans genèse sociale ${ }^{2}$ ).

Il y a quelque vingt ou trente ans, m'étant placé devant l'échéance d'avoir à faire la théorie de ma pratique traduisante, j'ai eu la (bonne) surprise de découvrir que la littérature sur la question était alors d'une ampleur relativement limitée, à cette époque de la fin des années soixante. En français, il semblait qu'il n'y eût que les Problèmes théoriques de la traduction de Georges Mounin ${ }^{3}-$ mis à part la Stylistique comparée du français et de l'anglais de Jean-Paul Vinay et Jean Darbelnet, et quelques «classiques» comme Valery Larbaud... De fait, comme on sait, cet ouvrage a longtemps fait autorité, en partie justement parce qu'il a été longtemps à peu près le seul qui fût connu; mais ces derniers temps la tendance est à le considérer comme "dépassé». À ce propos, il convient de reprendre une remarque épistémologique d'ordre général que j'ai faite en ce qui concerne la récente réédition de mon propre livre ${ }^{4}$ et qui vaut aussi pour celui de Mounin.

En vertu d'une idéologie - qui, comme beaucoup de bonnes choses et... de moins bonnes, nous vient en Europe d'outre-Atlantique -, il semblerait qu'à peine quelques années après sa parution, un livre fût «dépassé» et dût disparaître des bibliographies en sciences humaines comme des librairies. $\grave{A}$ en croire d'aucuns, les livres vieilliraient en sciences humaines aussi

2. René Lourau, Sociologue à plein temps (Paris, L'Épi, 1978).

3. Georges Mounin, les Problèmes théoriques de la traduction (Paris, Gallimard, 1963; rééd. 1976: coll. «Tel», $n^{\circ}$ 5).

4. Traduire: théorèmes pour la traduction (Paris, Gallimard, coll. «Tel», $\left.n^{\circ} 246,1994\right)$. La première édition était parue en 1979 , dans la Petite Bibliothèque Payot $\left(n^{\circ} 366\right)$; cette seconde édition en est la reprise, augmentée d'une préface ( $\mathrm{pp}$. v-xxi), avec une pagination identique (pour le corps du texte) et quelques coquilles en moins. (Je ferai désormais référence à ce livre en l'appelant Théorèmes...) 
vite qu'en sciences exactes, ce qui, à l'évidence, est erroné et procède de cette idéologie qu'on appelle tout simplement depuis plus d'un siècle le positivisme. L'idée est qu'il n'est de connaissance que scientifique, et que les sciences humaines ne seraient crédibles qu'autant qu'elles s'identifient aux sciences exactes, qu'elles les singent!

Il y aurait beaucoup à dire sur cette idéologie philosophiquement simpliste et fausse, et dont il n'est pas certain qu'elle ne recouvre pas les intérêts corporatistes plus troubles d'une sorte de syndicat des nouveaux venus de la production intellectuelle, dans le cadre d'un contexte de concurrence universitaire (publish or perish) et d'inflation éditoriale... Nous nous contenterons de rappeler que les sciences humaines constituent une culture spécifique de la modernité - une "troisième culture» pour ainsi dire, à côté de la «culture» traditionnelle et de ce qu'il faut bien appeler la culture scientifique $^{5}$ - et que les travaux auxquels elles donnent lieu ne sont (heureusement!) pas soumis au même rythme d'obsolescence que les publications émanant de la recherche scientifique (stricto sensu). Voit-on qu'il faille renoncer à lire Freud et Piaget, Marx et Max Weber, Saussure et Jakobson, etc., au motif qu'ils seraient «dépassés ${ }^{6} » ?$

Il est vrai que les auteurs qui viennent d'être cités en exemple constituent pour les sciences humaines considérées ce qu'il est convenu d'appeler des pères fondateurs; et qu'il n'est pas moins certain que la recherche et la réflexion «avancent» ou «progressent»

5. Voir mon étude «Pour une philosophie de la traduction», Revue de métaphysique et de morale, $\mathrm{n}^{\circ} 1$ (1989), p. 14 et passim. Cette idée des sciences humaines comme troisième culture rejoint celle qui a été exposée depuis par Wolf Lepenies, les Trois Cultures. Entre science et littérature l'avènement de la sociologie (Paris, Éditions de la Maison des Sciences Humaines, 1990; trad. fr. H. Plard).

6. Ces deux derniers alinéas reprennent ma nouvelle préface aux Théorèmes..., p. vi sq. 
aussi à leur façon dans ces domaines. Il reste qu'en sciences humaines - et c'est une spécificité épistémologique de ces disciplines qui, justement, les désigne comme une troisième culture - les grands auteurs, ou même seulement les bons auteurs, gardent une bonne part de leur actualité et font périodiquement l'objet de relectures, voire de redécouvertes. En cela, on le voit, ils ne se distinguent guère des auteurs de la tradition philosophique. Au reste, Marx ou Freud, par exemple, ne sont-ils pas tout simplement des philosophes? et puis, pour ce qui nous occupe ici, il y a la relation particulière entre linguistique et philosophie.

Quoi qu'il en soit, sans faire de Mounin un grand philosophe, je dirai que son œuvre reste à lire. Aussi l'archéologie dont j'ai annoncé ici l'esquisse ne veut pas dire qu'il n'y aurait plus qu'un intérêt «archéologique» à ses travaux, qui seraient comme les vestiges d'une préhistoire de la traductologie... Comme s'il convenait d'oublier Mounin - un peu comme il conviendrait d' (uoublier Foucault», si l'on en croit un Jean Baudrillard'? Tel n'est pas du tout bien sûr le point de vue qui est le mien.

Pour ma part, je tiens qu'il convient de voir en Mounin l'un des «pères fondateurs» de ce qui allait s'appeler plus tard la traductologie; et ce, même s'il a récusé par avance le mot lui-même. En tout cas, ses travaux ont eu pour ma propre recherche sur la traduction un rôle inaugural. C'est un peu ce qu'indiquait le rapprochement identifiant ses Problèmes théoriques de la traduction et mes Théorèmes pour la traduction que je me suis permis d'esquisser plus haut; et de fait c'est aussi, plus précisément, au sens d'une auto-archéologie que la traductologie fait ici l'objet d'une «esquisse archéologique».

\section{2. À partir de Mounin}

Par rapport à Mounin, je m'inscris dans un rapport intellectuel de filiation critique (ou dialectique), désignant ainsi un certain mode de

7. Jean Baudrillard, Oublier Foucault (Paris, Galilée, 1977). 
relation intellectuelle où je distinguerai trois aspects. D'abord, pour ce qui nous concerne et en raison de ce qui vient d'être dit, c'est le premier auteur que j'aie lu sur les problèmes théoriques de la traduction; et, bien que notre relation soit restée exclusivement livresque (et épistolaire), c'est lui qui m'a ouvert à ce type de réflexion. Ensuite, bien sûr, j'en ai fait la critique; et j'ai pris appui sur ce travail de critique pour développer ma propre réflexion sur la question. Mais, pour finir, je dirai que je continue à me situer dans le prolongement de ses travaux et à assumer son héritage dans la mesure où, malgré mes nombreuses critiques à son égard, je partage avec lui un certain nombre de positions fondamentales touchant la philosophie linguistique qu'implique toute théorie de la traduction, et notamment la critique du littéralisme ou la démystification du signifiant... Alors que par rapport à un Henri Meschonnic - dont j'ai été aussi l'élève, pour l'avoir lu, mais aussi pour avoir suivi ses enseignements - il m'est apparu que sur l'essentiel, justement, nous nous opposions ${ }^{8}$.

Il est à noter qu'en cette affaire, comme il semble que ce doive être la règle dans le contexte qui nous occupe, la ligne de démarcation entre philosophie et sciences humaines, en l'occurrence entre philosophie et linguistique, n'est plus très nette. Non seulement ce type de filiation intellectuelle est en effet quelque chose de fréquent dans la tradition philosophique mais, ainsi qu'il vient d'être indiqué, cela concerne aussi le contenu. Au reste, je reprendrai ici une formule qu'affectionnait mon regretté maître Ferdinand Alquié, qui tenait qu' «en philosophie, on ne réfute que ce qu'on a mal compris», avec ce qu'une maxime pédagogique de ce type peut comporter d'exagération rhétorique; et je l'appliquerai au contexte de la théorie de la traduction pour ainsi dire par synecdoque, dans

8. C'est déjà ce qu'a marqué la polémique que j'allais avoir avec Henri Meschonnic - ou, plus précisément, dont il allait prendre l'initiative -(Jean-René Ladmiral et Henri Meschonnic, «Poétique de.../Théorèmes pour... la traduction», Langue française), controverse opposant ceux que j'ai appelés les «sourciers» (comme Henri Meschonnic) et les «ciblistes» (comme Georges Mounin et moi-même), cf. infra. 
l'idée qu'en somme la philosophie est une synecdoque de toute vie intellectuelle et qu'à ce titre les leçons qu'on peut en tirer s'appliquent à la traductologie.

Dans le même esprit - et à la différence d'un Henri Meschonnic qui, à longueur de pages, réfute d'innombrables auteurs - j'ai longuement et à plusieurs reprises critiqué Mounin, non pas pour le «réfuter» (ce qui eût totalement manqué d'intérêt), mais pour approfondir des problèmes qu'il posait et, en en critiquant ce qui m'en semblait caduc, en prolonger les enseignements. Lui-même ne s'y est pas trompé, qui a consacré quelques études à mes propres travaux...9

Peut-être suis-je même allé un peu loin dans la critique quand en $1972^{10}$, reconnaissant à Problèmes théoriques de la traduction la qualité d'être «un excellent 'cours de linguistique générale'» (ce qu'il est, quoique un peu daté), je contestais qu'on pût en revanche y trouver véritablement les éléments d'une théorie de la traduction. Depuis ce péché de jeunesse, $j$ 'ai fréquemment pris appui sur ce livre pour mes divers enseignements de théorie de la traduction (d'autant qu'entre temps une édition de poche en facilitait

9. Ainsi ai-je été, très tôt, l'auteur le plus vivement et le plus abondamment critiqué par lui, par exemple, dans «la Traduction en 1975 (Un bilan français)», Linguistique et traduction (Bruxelles, Dessart et Mardaga, coll. Psychologie et sciences humaines, $\mathrm{n}^{\circ} 60,1976$ ), p. 205 sqq. Plus récemment, après la parution des Théorèmes..., il m'a même fait l'honneur d'une étude détaillée: «La Traductologie de Jean-René Ladmiral», Écritures à Maurice-Jean Lefebvre, A. Ningelgrün et A. Nysselholc, dir. (Bruxelles, Éditions de l'Université de Bruxelles, 1983), pp. 141-155.

10. «Introduction» au numéro de Langages (Didier-Larousse) que j'ai dirigé sur la Traduction $\left(\mathrm{n}^{\circ} 28\right.$, décembre 1972), p. 4; reprise dans Théorèmes..., p. 260 et passim. 
l'acquisition par les étudiants "), non seulement parce que j'y vois l'occasion d'une «remise à niveau» (au moins partielle) en linguistique, mais aussi parce qu'il y a matière à en dégager des éléments de réflexion sur la pratique traduisante, dès lors qu'on en fait une lecture critique. Peut-être est-ce à travers même les déficits qu'y décèle la critique (théorique et pratique) qu'il prend toute sa valeur de formation intellectuelle. C'est au demeurant le bon usage de la littérature en sciences humaines, sinon même en philosophie, que de construire sa compétence sur l'assimilation sélective, et en quelque façon «cannibalique», de ceux qui nous ont précédés dans la discipline.

Ainsi ai-je construit ma théorie de la traduction à partir de Georges Mounin, au double sens où «partir de» ses travaux, c'était en venir, commencer avec eux, mais c'était aussi n'y point rester, aller au-delà : c'est ce qu'entend marquer ici le libellé même de mon titre ${ }^{12}$.

\section{Arguments et objections}

Fondamentalement, j'ai fait - et je fais - trois ou quatre critiques au discours que développent les Problèmes théoriques de la traduction. La première et la principale est de s'être enfermé dans ce que j'ai appelé la problématique de l'objection préjudicielle, c'est-à-dire de s'être posé une question séculaire et «métaphysique» à laquelle il n'y a guère à proprement parler de réponse - à savoir: La traduction est-elle possible? - et donc de s'y être «empêtré». Il y a là un archaïsme théorique qui est en contradiction avec la profession de foi affichée d'une modernité et d'une rigueur méthodologique qu'est censée conférer à ce discours son allégeance à la linguistique (à la

11. C'est même l'un des tout premiers volumes publiés dans la collection «Tel» $\left(n^{\circ} 5\right)$, en 1976.

12. Cette filiation intellectuelle, $j$ 'en ai eu conscience et je l'ai assumée très tôt: cf. Théorèmes..., p. 88. 
linguistique d'alors). De fait, cette problématique-là traverse toute l'œuvre traductologique de Mounin; et ce, dès les Belles Infidèles ${ }^{13}$, antérieures aux Problèmes théoriques de la traduction et de facture plus littéraire, mais où on trouvera déjà l'essentiel des arguments développés par l'auteur.

Point n'est besoin de reprendre ici dans le détail mes critiques sur cette question ${ }^{14}$; je m'en tiendrai à n'en indiquer que l'argument ou, plus précisément, le squelette de l'argumentation et son issue. D'une façon générale, le discours que tient Mounin est le plus souvent d'ordre polémique. Dans les Belles Infidèles, il reprend les trois types d'«arguments contre la traduction» (polémiques, historiques et théoriques) qu'indiquait déjà Joachim du Bellay dans sa Défense et illustration de la langue française.

Les prétendus «arguments polémiques» ne consistent qu'à exciper de l'existence de mauvais traducteurs pour instruire un procès contre la possibilité de la traduction elle-même. On conviendra, avec Pierre Vidal-Naquet, qu' «il faudrait quand même tuer le général Staff»! Je fais ici allusion à un billet piquant qu'avait publié l'historien dans le quotidien parisien du soir, où il brocardait la bévue de traducteurs qui prennent non pas Le Pirée, mais le general Staff (en anglais: l'état-major) pour un homme, un général $^{15} \ldots$ Mais $\mathrm{j}$ 'ai indiqué aussi que l'argument se retourne

13. Georges Mounin, les Belles Infidèles (Paris, Cahiers du Sud, 1955). Ainsi que je l'ai affirmé maintes fois (cf. Théorèmes...,p. 87 et passim), je ne suis pas éloigné de penser que c'est là son meilleur livre, à certains égards. Il faut donc se réjouir de sa récente réédition, en 1994, aux Presses universitaires de Lille, dans la collection Étude de la traduction.

14. C'est tout l'objet du troisième chapitre des Théorèmes..., pp. 85-114.

15. Pierre Vidal-Naquet, «Bévues de traduction - Tuer le général Staff), Le Monde (18 janvier 1974), p. 16. Je cite in extenso ce petit texte dans les Théorèmes..., p. 93 sq. 
comme un gant, que l'argument «polémique» contre les mauvais traducteurs est en même temps un contre-argument pour la traduction dans la mesure où il atteste $a$ contrario l'existence de bons traducteurs ${ }^{16}$.

Quant aux «arguments historiques», ils reviennent à resituer la problématique de l'objection préjudicielle dans le contexte historique qui était celui de du Bellay et, au-delà, à faire le «procès historique d'une langue traductrice donnée ${ }^{17}$ ». À vrai dire, un tel argument «historique» est de tous les temps; et, touchant le dernier point, Mounin le soulignera lui-même, ailleurs, d'une formule heureuse: «richesse merveilleuse de toutes les langues de départ, pauvreté incurable de toutes les langues d'arrivée ${ }^{18}$ ')! $\mathrm{Au}$ demeurant, ces quelques mots résument à eux seuls une bonne part de l'idéologie spontanée touchant le langage (et les langues) dont la traduction est le révélateur - tant il est vrai que «la traduction a le pouvoir de nous rendre bêtes!» dirai-je, au sens où elle fait trop souvent oublier les quelques évidences fondatrices que nous a enseignées la linguistique. C'est ce que j'appellerai l'«illusion traductive», dont une Dialectique de la traduction (au sens quasi kantien que pourrait prendre cette expression) aura à faire la critique $^{19}$.

Sans épuiser cette question des rapports entre «traduction et histoire», il conviendra d'indiquer au passage encore au moins deux

16. Ibid., p. 93.

17. Les Belles Infidèles, p. 19.

18. Georges Mounin, article «Traduction», André Martinet, dir., la Linguistique. Guide alphabétique (Paris, Denoël-Gonthier, 1969; rééd. 1972; coll. Guides alphabétiques Médiations), pp. 376. Ce petit texte, ainsi que plusieurs autres, a été repris par Mounin dans son livre Linguistique et traduction, pp. 71-76.

19. Je développerai ce point dans une prochaine étude, dans le cadre d'une discussion des thèses d'Antoine Berman. 
remarques. D'une part, s'il est vrai, comme le rappelle Mounin, que c'est pour des raisons historiques et pour renforcer, pour «défendre et illustrer» le français que du Bellay argumente contre la traduction qui, au même titre que le latin et le grec (et sans doute aussi l'italien, voire l'espagnol), menaçait le français encore in statu nascendi, il est tout aussi indéniable qu'inversement la traduction peut être l'acte de naissance d'une langue, comme on voit pour l'allemand avec la Bible de Luther et comme l'indique aussi, pour le français lui-même, Roger Zuber, mit en evidence, à propos de ce genre littéraire qu'était la littérature historique, que la traduction se constitue elle-même comme un genre, au role décisif dans la consolidation de notre langue ${ }^{20}$. De par la langue «au service de» laquelle il arrive qu'elle se mette, la traduction peut ainsi être indirectement fondatrice d'identité, d'identité linguistique, mais aussi d'identité nationale, voire d'identité religieuse ${ }^{21}$.

D'autre part, et surtout, l'objection préjudicielle d'intraduisibilité perd son caractère d'en-soi «métaphysique», flottant en dehors du temps, dès lors que sera réhistoricisée la traduction, ainsi que le travail qu'elle implique sur la langue et la culture d'une société. Comme y insiste, à juste titre, Henri Meschonnic, «l'intraduisible est [social et] historique, [et] non métaphysique $\left.{ }^{22}\right)$. J'en suis bien d'accord et cette indéniable vérité est l'une des raisons qui me font récuser la problématique de l'objection préjudicielle, mais ce n'est pas la seule, ni peut-être

20. Roger Zuber, les "Belles Infidèles" et la formation du goût classique. Perrot d'Ablancourt et Guez de Balzac (Paris, Armand Colin, 1968).

21. C'est là une perspective que j'ai abordée dans «Pour une théologie de la traduction», TTR, III(2) (2 sem. 1990), pp. 121-138.

22. Henri Meschonnic, «Poétique de la traduction», Pour la poétique II (Paris, Gallimard, coll. Le Chemin, 1973), pp. 309, 411... 
même la principale. Fondamentalement, ce qui est là en cause, c'est le concept même de traduction dont la définition fait problème ${ }^{23}$.

\section{Dimension socio-historique de la traduction}

À la différence d'Henri Meschonnic, je ne pense pas qu'il faille induire de l'intraduisibilité une sorte d'ontologie holiste où la spécificité "iidiomatique» de la langue se trouve "dédifférenciée», dissoute dans le socio-historique, au nom d'un sociologisme marxisant et d'un matérialisme historique essentiellement programmatique, et donc idéologique. Au mieux, la façon incantatoire de Meschonnic d'assigner les problèmes de la traduction à une théorie du langage en général et cette dernière à l'horizon global d'une théorie de la société et de l'histoire est, sur le plan théorique, une manière de «botter en touche ${ }^{24} \|$; et ce, même s'il est indéniable que l'auteur fait preuve d'une réelle finesse dans les analyses critiques et même polémiques qu'il fait des traductions des autres ${ }^{25}$.

Cela fait penser à l'usage qui est fait du concept de social dans le discours d'une certaine scolastique marxiste, où ce concept a une fonction plus apologétique que méthodologique, et une teneur plus ontologique que proprement «matérialiste» et «scientifique». En l'occurrence, ledit «social» s'oppose au sociologique qui, lui, irait

23. Voir les Théorèmes..,p. pviii sq., et «Traduire, Qu'est-ce à dire? Phénoménologies de la traduction» (Meta, à paraître en septembre 1995).

24. C'est tout l'enjeu de la polémique qui nous a opposés, et qui a été signalée à la note 8. Au-delà - au-delà de ce texte de circonstance, mais qui est tout à fait révélateur, ou «symptomal» -, la réflexion d'Henri Meschonnic participe de cette ontologie du social, voire d'une ontologie du signifiant.

25. Un exemple parmi tant d'autres: Henri Meschonnic, «Traduire la Bible, de Jonas à Jona», Langue française, $\mathrm{n}^{\circ} 51$ (septembre 1981), p. 35 sqq. 
dans le sens d'un travail «sur le terrain» des réalités qu'on prendrait la peine d'étudier, au lieu de présupposer que la «position juste» qu'on occupe permet de s'en dispenser et, disposant de la science infuse, de s'instituer en tribunal épistémologique multipliant les anathèmes. Et l'on sait combien le discours d'Henri Meschonnic est polémique! Pour ainsi dire, il l'est par nature; même s'il est vrai que ses travaux plus récents tendent à défendre des positions qui semblent parfois plus nuancées...

La langue est la langue! et les axiomes fondateurs de la linguistique nous apprennent quelque chose de sa spécificité, quand bien même il n'est que trop évident que la langue est aussi une réalité historique de la société où, dirons-nous (pour rester dans la même isotopie discursive...), elle fonctionne à la fois comme «superstructure» sémantique et stylistique, et comme «base» ou infrastructure signifiante, idiomatique et communicationnelle. Cela dit, il est vrai que, jusqu'à présent, je n'ai pas encore développé ma propre recherche dans le sens de la dimension sociologique et sociolinguistique de la traduction ${ }^{26}$.

$\grave{A}$ vrai dire, il est encore une déviation que peut induire la légitime réhistoricisation de la traduction. Une certaine relativisation de la langue et en particulier de la langue-cible, qui est donc une réponse à l'objection préjudicielle, est fondée dès lors qu'on s'en tient à prendre en compte la diachronie des langues en tant qu'elle est porteuse d'innovation néologique et qu'en somme on récuse là l'essentialisme esthétisant et conservateur des puristes ${ }^{27}$. On peut assister, certes, à un certain dégel du français depuis quelques années et on ne songera qu'à s'en féliciter vivement: tout particulièrement les traducteurs qui, on le sait, ne se trouvent que trop souvent confrontés à l'échéance d'une obligation néologique. Des textes jusqu'alors intraduisibles deviennent traduisibles. De plus, il est

26. C'est même l'un de ces «chantiers» qui restent ouverts à des travaux à venir.

27. Théorèmes..., p. 202. 
avéré que la traduction est une des voies les plus productives de néologie dans les langues.

Cela dit, quand Henri Meschonnic, mais aussi le regretté Antoine Berman, ou un Walter Benjamin en viennent à prôner, après Pannwitz, que la norme de la langue-cible soit violée pour être pliée aux exigences de la langue-source, ils méconnaissent ce qui fait la nature même, le "propre» ('̌oios) de ce que c'est qu'une langue, justement appelée un idiome - pour aller dans le sens d'une réduction «socio-historiciste» de la langue telle que je viens d'en faire la critique chez $\mathrm{H}$. Meschonnic. Le caractère éminemment fallacieux de cette «logique» ne m'est certes pas apparu d'emblée $^{28}$. Ce n'est qu'un peu plus tard ${ }^{29}$ que j'ai pris la mesure du problème dans toute l'ampleur de ses enjeux. La question du littéralisme qui se trouvait posée là, et qui est une question immémoriale, allait m'occuper dans ce qui allait être un second temps de ma recherche, plus explicitement philosophique: $c$ 'est ainsi que j'en suis venu à camper l'opposition entre sourciers et ciblistes $^{30}$ et à en problématiser les implications, voire les «impensés ${ }^{31}$ ».

28. Ainsi, dans les Théorèmes..., p. 173 sq, la profondeur de l'opposition (et la virulence des polémiques à venir) n'est-elle encore que pressentie.

29. À partir de 1981: cf. «Entre les lignes, entre les langues», dans le cadre du numéro Walter Benjamin de la Revue d'esthétique (nouvelle série), $\mathrm{n}^{\circ} 1$ (1981), pp. 67-77.

30. «Sourciers et ciblistes», Revue d'esthétique, $\mathrm{n}^{\circ} 12$ (1986), pp. 33-42.

31. Il y a là la problématique fondamentale d'une philosophie de la traduction, qui fournira la matière du prochain livre et qui, de fait, est déjà le fil rouge qui traverse différents articles que j'ai publiés depuis quelques années: «la Traduction proligère? Sur le statut des textes qu'on traduit», Meta, XXXV(1) (mars 1990), pp. 102-118;

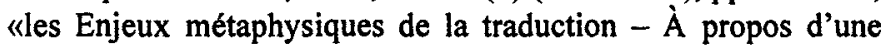




\section{Rapport à la linguistique}

Pour en revenir à Mounin, on a vu que je fais la critique de la problématique de l'objection préjudicielle, en regrettant que ce soit sous ses auspices qu'il ait développé l'essentiel de ses réflexions sur la traduction, d'abord dans le discours littéraire des Belles Infidèles, puis dans le discours linguistique des Problèmes théoriques de la traduction. Ma seconde critique à ce dernier livre (ainsi qu'à la plupart de ceux qui suivront) porte sur le caractère restrictivement linguistique, justement, et même «linguisticiste» de son propos.

Dès le premier chapitre, l'auteur pose la question qui servira d'intitulé au deuxième chapitre et qui est en quelque sorte le programme de l'ensemble du livre: «L'étude scientifique de l'opération traduisante doit-elle être une branche de la linguistique? ${ }^{32}$ »). En réalité, c'est là une question rhétorique, à laquelle il est implicitement très clair que la réponse sous-entendue est oui! et elle est intimement liée à la problématique de l'objection préjudicielle dans la mesure où, pour Mounin, le problème posé sera de savoir si les grandes théories de la linguistique structurale - pour des raisons historiques évidentes, ce sont les seules auxquelles il puisse se référer - sont compatibles avec l'existence séculaire de la pratique traduisante. Autrement dit: les théories linguistiques en vigueur (naguère) permettent-elles de rendre compte de la traduction?

D'abord - et ce n'est pas un mince paradoxe - il y a quelque chose d'apologétique dans l'argumentation que déploie ce plaidoyer au service d'un engagement épistémologique en faveur de

critique de Walter Benjamin", le Cahier du Collège International de Philosophie, $\mathrm{n}^{\circ} 6$ (1988), pp. 39-44; cf. supra notes 21, 29, 30.

32. Les Problèmes théoriques de la traduction, p. 10. 
la discipline linguistique ${ }^{33}$. Surtout: il m'est apparu très tôt que c'était accorder à la linguistique trop de crédit que d'attendre d'elle qu'elle puisse, à elle seule, nous fournir une théorie de la traduction $^{34}$. Ainsi ai-je, par exemple, critiqué Mounin (encore) pour avoir voulu définir la traduction comme une «opération linguistique ${ }^{35} \|$ (comme $s^{\prime}$ il $y$ avait là une avancée théorique décisive!) alors que, tant qu'à faire, je pense qu'il conviendrait d'aller plus loin et d'élargir le concept aux dimensions de ce que j'ai préféré appeler une pratique sémiotique ${ }^{36}$. Plus généralement, c'est le statut linguistique lui-même de la théorie de la traduction que j'allais être conduit à mettre en cause; il m'est apparu en effet qu'il y avait lieu de situer la traductologie dans la perspective d'une ouverture résolument interdisciplinaire qui mette à contribution l'ensemble des sciences humaines et de ce qu'il est traditionnellement convenu d'appeler «les Humanités», tout en faisant porter l'accent sur le triptyque: linguistique, psychologie et philosophie $^{37}$.

33. Autant le discours apologétique d'un engagement idéologique, comme c'est le cas chez Henri Meschonnic (cf. supra et infra), m'apparaît contestable, autant était-il dans «l'air du temps» des années soixante... Chez Mounin, le paradoxe est d'une apologétique épistémologique, ce qui fait figure de contradictio in adjecto.

34. Théorèmes..., p. 8 et passim, notamment «Sourciers et ciblistes», p. 33 sq., etc.

35. Les Problèmes théoriques..., p. 10 et passim.

36. Théorèmes..., p. 208.

37. Théorèmes...,pp. ix sqq. et xxi, ainsi que «Pour une philosophie de la traduction"), p. 10. 
Logiquement, Mounin regrettait que la traduction fût absente des traités et manuels de linguistique ${ }^{38}$; en sorte que son plaidoyer pour une théorie linguistique de la traduction entend inaugurer une tradition. Mais s'il est vrai qu'il a été l'un des premiers, il n'était pas le premier; et il se reconnaît lui-même quelques prédécesseurs, comme Jean-Paul Vinay et Jean Darbelnet ${ }^{39}$, dont il fait grand cas. La stylistique comparée, dès lors incarnée par ces deux auteurs (aux côtés de Malblanc ${ }^{40}$, que Mounin omet de $\operatorname{citer}^{41}$ ), a fait depuis l'objet de diverses critiques «dans le milieu». D'une façon générale, la plupart des théoriciens de la traduction et autres traductologues s'accordent à considérer cette approche a fortiori comme «dépassée» (cf. supra). Sur ce point, j'ai adopté une position nuancée; et, comme souvent, je ferai la distinction entre théorie et pratique.

\section{Pratique traductive et langues}

D'abord, pour J.-P. Vinay et J. Darbelnet, il est bien clair que ladite Stylistique comparée relève de la linguistique: d'entrée de jeu, par exemple, ils placent leur analyse des «notions de base» sous le patronage du Cours de linguistique générale de Ferdinand de

38. Les Problèmes théoriques..., p. 10. Et ce, bien que le discours linguistique, remarque-t-il après Jakobson, ne cesse de procéder implicitement à des opérations de traduction (ibid., p. 8). Cf. aussi Linguistique et traduction, p. 71, etc.

39. Jean-Paul Vinay et Jean Darbelnet, Stylistique comparée du français et de l'anglais, nouvelle édition revue et corrigée (Paris, Didier, 1973).

40. Alfred Malblanc, Stylistique comparée du français et de l'allemand. Essai de représentation linguistique comparée \& Étude de traduction (Paris, Didier, Bibliothèque de stylistique comparée, no II, 1961).

41. Les Problèmes théoriques..., p. 8; cet auteur est même absent de la bibliographie. 
Saussure $^{42}$. D'un point de vue théorique, il est vrai que leur travail, dont la première édition date de 1958, a beaucoup (vvieilli), à la fois parce qu'il fait fond sur une linguistique évidemment datée, qui est restée à l'écart des recherches récentes, et notamment parce que leurs analyses sont encore encombrées d'une sorte de psychologie linguistique qui tend à verser dans la psychologie des peuples (Völkerpsychologie) ${ }^{43}$.

Mais, au niveau propre de la pratique, j'ai toujours défendu ce livre ${ }^{44}$ au motif qu'il apporte une ample moisson d'exemples; et ce, quand bien même le propos théorique qui les sous-tend pourrait être "falsifié» par autant (ou presque) de contre-exemples! Si l'on en croit Auguste Comte, «on ne détruit que ce qu'on remplace»; et les travaux publiés depuis n'ont pas remplacé l'ouvrage de J.-P. Vinay et J. Darbelnet, ils sont venus s'y ajouter. J'irais même jusqu'à dire que, un peu comme pour le livre de Mounin lui-même, ce sont ses faiblesses théoriques qui l'indiquent pour l'enseignement, comme base sur laquelle puisse s'exercer la critique des étudiants dans le cadre des enseignements de traductologie de type réflexif tels que je les conçois (et les pratique) ${ }^{45}$.

42. Stylistique comparée du français et de l'anglais, p. 28 sqq.

43. Ainsi le français est-il plus «abstrait» et l'anglais plus "concret», etc. Cela dit, sur l'épistémologie de ces questions, cf. «Éléments d'imagologie franco-allemande», in Jean-René Ladmiral et Edmond-Marc Lipiansky, la Communicationinterculturelle(Paris, Armand Colin, 1989; rééd. 1991 et 1995), p. 228 sqq.

44. Voir encore «Technique et esthétique de la traduction», Actes des Journées européennes de la traduction professionnelle (UNESCO, Paris, 25-26 mars 1987): revue Encrages (Hachette) Université de Paris-VIII (Vincennes à Saint-Denis), $\mathrm{n}^{\circ} 17$ (printemps 1987), $\mathrm{p}$. 191.

45. Cela fera l'objet d'une prochaine étude dans le cadre d'une Didactique de la traduction, autre «chantier» que j'ai laissé ouvert jusqu'à présent. 
À vrai dire, la critique que je fais de la Stylistique comparée est beaucoup plus fondamentale; et elle m'a aidé a contrario à définir le projet traductologique qui est le mien. L'apparent privilège de la pratique par rapport à la théorie que je viens d'invoquer pour la défense de J.-P. Vinay et J. Darbelnet n'est pas une sorte de rechute dans le «poujadisme» practiciste auquel cèdent tant de traducteurs et dont j'ai déjà fait la critique dans Théorèmes...; il renvoie à l'articulation entre théorie et pratique, pour ce qui est du domaine spécifique de la traduction, telle que je me suis efforcé de le définir dans le cadre d'une «épistémologie de la traduction» sur laquelle il y aura lieu de revenir ${ }^{46}$. À un niveau non pas encore épistémologique mais méthodologique, c'est le statut même de la linguistique au sein de ce mode spécifique de traductologie représenté par la Stylistique comparée qui est en cause.

Ce que J.-P. Vinay et J. Darbelnet ont en commun avec les travaux, linguistiquement plus convaincants, qui ont été publiés depuis dans la même direction - comme ceux de Jacqueline Guillemin-Flescher ${ }^{47}$ ou de Michel Ballard ${ }^{48}$, ou encore de Peter

46. Dans le prolongement des quelques études déjà effectuées: «Philosophie de la traduction et linguistique d'intervention», Lectures (Bari, Dedalo), $\mathrm{n}^{\circ}$ 4-5 (août 1980), pp. 11-41; «Quelles théories pour la pratique traduisante?», la Traduction. Actes des Rencontres autour de la traduction (Paris, B.E.L.C., 1986), pp. 145-166; "Technique et esthétique de la traduction», loc. cit; «Épistémologie de la traduction», Reiner Arntz, dir., Textlinguistik und Fachsprache. Akten des Internationalen AlLA-Symposions Hildesheim (13-16 avril 1987), (Hildesheim, Georg Olms Vlg., 1988), pp. 35-47.

47. Jacqueline Guillemin-Flescher, Syntaxe comparée du français et de l'anglais. Problèmes de traduction (Gap, Ophrys, 1981).

48. Michel Ballard, la Traduction: de l'anglais au français (Paris, Nathan, coll. Nathan-Université, 1987). 
Blumentha ${ }^{49}$, etc. - c'est de s'appuyer sur ce qu'on pourrait appeler en quelque sorte une «linguistique de la langue»! D'où une traductologie de la langue et une traductologie linguistique dont je fais la critique, ce qui me conduit à définir le programme d'une approche que je crois plus adéquate aux réalités de la pratique traduisante. Ma critique est double: d'une part, la traduction n'est pas une affaire de langues; et, d'autre part, il m'est apparu de plus en plus clairement que l'essentiel de la traductologie n'est pas restrictivement linguistique.

D'abord, en traduction, les principales difficultés ne sont pas des difficultés de langue(s) - c'est un point sur lequel, avec d'autres, j'ai insisté en plusieurs occasions ${ }^{50}$. Certes "pour traduire, il faut connaître des langues", comme le reconnaît Edmond Cary ${ }^{51}$, mais cette condition nécessaire est très loin d'être suffisante. Au-delà des difficultés de langues (des difficultés liées à la maitrise de la langue maternelle, plus encore sans doute qu'à celle des langues étrangères...), il y a bien sûr le problème de la terminologie, des terminologies, et celui d'une certaine compétence minimale à acquérir concernant le domaine dont traitent le texte à traduire et la terminologie qu'il met en œuvre; et puis il y a les difficultés

49. Peter Blumenthal, Sprachvergleich Deutsch-Französisch (Tübingen, Niemeyer, Romanistische Arbeitshefte, $n^{\circ} 29,1987$ ).

50. «La Traduction: philosophie d'une pratique», les Gens du passage, Christine Pagnoulle, dir. Université de Liège, 1992 (L3 - Liège Language and Literature), p. 128 sq. ou encore «l'Ordinateur est une vraie machine à écrire», l'Environnement traductionnel. La station de travail du traducteur de l'an 2001 (Actes du colloque de Mons, 25-27 avril 1991), André Clas et Hayssam Safar, dir. (Montréal, AUPELF/Presses de l'Université du Québec, 1992), p. 329 sq., etc. J'y reviendrai dans le cadre de la didactique de la traduction précédemment annoncée (cf. note 45) et d'une psychologie du traducteur, à constituer.

51. Edmond Cary, Comment faut-il traduire? (rééd.), introd. Michel Ballard (Lille, Presses universitaires de Lille, 1986), p. 29. 
spécifiques liées à l'expérience et à la maîtrise des processus psycho-linguistiques propres à l'opération traduisante elle-même ${ }^{52}$.

C'est là proprement la compétence traductive, dont l'analyse scientifique relève $d u$ uchantier» que nous laissons ouvert sur la psychologie du traducteur, mais qui, parallèlement, devra faire l'objet d'une pédagogie dans le cadre de la formation des traducteurs ${ }^{53}$. $\dot{A}$ ce propos, notons au passage que ce n'est pas tout à fait le même problème que celui d'un "cinquième objectif» qu'il est possible d'assigner à l'enseignement des langues (une

52. J'ai commencé à traiter de ce cinquième type de difficultés et de l'«alchimie» psycho-linguistique de la traduction dans «Traduction et ambiguïté», Brises (Bulletin de recherches sur l'information en sciences économiques, humaines et sociales), $\mathrm{n}^{\circ} 7$ (octobre 1985), p. 59 sqq. et dans «l'Ordinateur est une vraie machine à écrire», loc. cit., p. 330 sqq.; j'y reviens dans l'étude intitulée "Que le traducteur est un réécrivain", Actes de la Journée d'études internationales, (Ré)écritures littéraires et utilitaires, organisée par Gertrud Gréciano à l'Université des Sciences Humaines de Strasbourg (2 mai 1994), à paraître dans les Nouveaux Cahiers d'Allemand (publiés près l'Université de Nancy).

53. À vrai dire, il s'agira moins de mettre en place un apprentissage, à proprement parler, qu'une sensibilisation-dans la mesure où la compétence traductive est une praxie globale qui est déjà là au départ et qu'il suffit de l'exercer - tel est du moins le postulat dont je pars. Il reste dès lors à définir le type de dispositif pédagogique le plus adapté. Tout cela fera l'objet d'une prochaine étude, faisant le bilan de mon expérience de formateur dans ce domaine; cf. déjà mon étude intitulée «Traduction philosophique et formation des traducteurs", Translation Theory and its Implementation in the Teaching of Translating and Interpreting (Tübingen, G. Narr Vlg., Tübinger Beiträge zur Linguistik, $n^{\circ} 247$, 1983), pp. 231-240. 
«cinquième skill $\left.{ }^{54}\right)$ ). Le premier problème est d'ordre psychologique, alors que le second relève de la didactique de la traduction.

Comme on sait: plus encore qu'une connaissance des langues, c'est une maîtrise psychologique de la compétence traductive et l'accumulation culturelle d'un bagage cognitifis que présuppose un exercice efficace de l'activité de traduction. Au-delà des échéances de la pratique, l'accent mis exclusivement sur une «linguistique des langues» interdit de prendre toute la mesure des problèmes de la traduction sur le plan théorique. Il n'existe pas d' "axes paraphrastiques" permettant de passer d'une langue à l'autre langue ${ }^{56}$. Pourtant, l'illusion est tenace qui voudrait qu'on puisse passer d'un énoncé d'une langue à un énoncé d'une autre langue, supposé synonyme, par une succession de transformations linguistiques minimales accumulées, sans solution de continuité (ainsi que semble le présupposer Michel Ballard, par exemple). En totale contradiction avec cette idée d'un continuum linguistique, qui ne correspond en rien à la réalité des langues, l'expérience de traduire est celle de ce que j'ai appelé «le salto mortale de la déverbalisation ${ }^{57}$ », à quoi viennent s'ajouter le «bricolage» et l'à-peu-près d'un ajustement contextuel et stylistique ${ }^{38}$.

54. C'est la thèse que je défends dans «Pour la traduction dans l'enseignement des langues: 'version' moderne des Humanités», les Langues modernes, $\mathrm{n}^{\circ} 1$ (1987), p. 13.

55. C'est ce que je visais (sub specie linguae) à travers le concept de "périlangue», Théorèmes..., p. 61 et passim.

56. Modèle critiqué dans Théorèmes..., p. 124 sqq.

57. «L'Ordinateur est une vraie machine à écrire», p. 331 et «Que le traducteur est un réécrivain» (cf. note 52).

58. Théorèmes..., pp. 125 et 127; et cf. «Épistémologie de la traduction», p. 43 sq. 


\section{Au-delà d'une maladie infantile}

Au terme de cette critique de la Stylistique comparée et d'un modèle linguistique transformationnel de la traduction, j'en suis venu à distinguer deux attitudes fondamentales opposées: il y a ceux que j'ai appelés les contrastivistes et ceux que j'ai appelés les traductologues $^{59}$. J'entends ici l'expression de «traductologues» au sens restreint où il s'agit de ceux qui partent d'une théorie de la traduction pour rejoindre la pratique traduisante, dans l'idée que «l'intendance linguistique suivra» - au lieu d'essayer de faire coller la description linguistique des deux langues concernées à la réalité du processus de traduction, ou l'inverse, ainsi que s'y efforcent les «contrastivistes».

Cette surestimation du facteur «langues» dans la traduction semblerait rapprocher ces derniers de ceux que j'appelés les sourciers ${ }^{60}$. Mais, en principe, lesdits contrastivistes connaissent trop bien les langues, dont ils ont fait leur spécialité, pour céder à l'illusion littéraliste: s'ils ont en commun avec les sourciers l'idée de mettre implicitement la subjectivité du traducteur entre parenthèses, leur sens de la langue est trop aiguisé pour que, dans la pratique, ils manquent à l'impératif de ce que j'appelle le «théorème» de dissimilation ${ }^{61}$.

Cela dit, on notera que les contrastivistes se comptent surtout parmi les anglicistes. Il y a là d'abord un effet de masse: parmi ces linguistes, spécialistes de langue étrangère, («les languistes» par opposition aux spécialistes de linguistique générale

59. Tel est l'enjeu de la controverse entre Michel Ballard et moi (14 mars 1986): «Quelles théories pour la pratique traduisante?» $\mathrm{p}$. 147 sqq.; cf. aussi «Épistémologie de la traduction», p. 39.

60. «Sourciers et ciblistes», pp. 33-42.

61. C'est, à mes yeux, le théorème principal qui doit être enseigné aux apprentis traducteurs; cf. Théorèmes..., pp. 161, 190, 218 et passim. 
ou «linguisticiens» selon ma terminologie), les anglicistes forment en tout état de cause les gros bataillons, le "grand frère» pour ainsi dire... Ce n'est pas au Canada, et encore moins au Québec, qu'on pourra ignorer cette donnée de fait. Cela tient sans doute aussi à des raisons d'ordre institutionnel: pour ce qui nous occupe, le marché linguistique anglais-français est sûrement le plus porteur quantitativement; en sorte que l'approche contrastiviste semble pouvoir être «amortie» dans ce contexte spécifique. Une raison très importante renvoie aussi à la problématique de la «distance linguistique» (William F. Mackey), de la distance inter-linguistique. En effet, du point de vue du français, l'anglais fait partie des langues proches: après les langues romanes, bien sûr, c'est encore une langue proche; alors que l'allemand fait figure déjà de langue "exotique»: ce serait la première des langues difficiles (deutsche Sprache schwere Sprache).

Au sein de la typologie à quatre termes que j'ai proposée des discours traductologiques, je classe les contrastivistes dans le cadre de la traductologie descriptive, à dominante linguistique, que j'ai stigmatisée comme la traductologie «d'hier ${ }^{62} »$.

$\mathrm{Au}$ vrai, l'erreur ne vient pas là de la linguistique elle-même, mais de la toute-puissance méthodologique qui lui est prêtée. Plus précisément, on fait l'hypothèse que la maîtrise linguistique des langues en présence suffira pour résoudre les problèmes de traduction. Autant et plus que d'une prétention épistémologique excessive de linguistes, il s'agit là d'une «déformation professionnelle» de pédagogues - selon le principe «Donnez-moi de la bonne linguistique, et je vous ferai de la bonne traduction»! Les leçons de la pratique montrent que les choses sont plus complexes, ainsi qu'il a été indiqué. En outre, on peut voir là une mouture de l'illusion philosophique assez générale qui consiste à prétendre résoudre les problèmes par un «déplacement» les

62. Voir note 59. La «traductologie» reprend ici un sens un peu moins restrictif, son sens normal. 
projetant au plan de l'éducation, en vertu de ce que j'ai appelé l'idéalisme pédagogique...

Conformément à l'esprit de la relation dialectique de filiation critique par rapport à Georges Mounin, j'ai moi-même situé ma réflexion traductologique dans le cadre de la linguistique, au départ. Que la traduction soit un objet relevant de la linguistique, c'est au demeurant l'opinion dominante, celle des linguistes et celle des chercheurs d'autres disciplines - même si ce n'est pas toujours celles des traducteurs eux-mêmes ${ }^{63}$... Il y avait des raisons relevant de l'histoire des sciences (humaines) à cette inscription de l'étude des problèmes de la traduction dans le cadre épistémologique du paradigme de la linguistique $e^{64}$. Le renouveau de la linguistique dans les années soixante, sous les auspices duquel nous avons situé à la fois les travaux de Mounin et notre propre intérêt pour la traduction, comportait certains aspects de ce qu'on pourra rétrospectivement qualifier comme une «maladie infantile».

La jeune «science linguistique» affichait une ambition programmatique dont on mesurait encore imparfaitement les limites et qui laissait donc une part d'incertitude dans la délimitation précise de ses champs d'études possibles et, plus encore peut-être, un certain «flou» touchant les contenus méthodologiques, dont il restait à explorer les ressources et les déficits. Corollairement, les prétentions épistémologiques de la linguistique d'alors débouchait sur un positivisme assez couramment répandu pour qu'il parût qu'en dehors de ses critères méthodologiques il n'y eût point de salut pour une étude "scientifique» d'objets langagiers comme la traduction. Sans avoir sur ce point la naïveté épistémologique à laquelle s'adonnaient allègrement bon nombre des linguistes de ma génération - et contre

63. Je ne reprends pas ici ce débat, dont Georges Mounin a pressenti l'essentiel dans sa discussion des positions d'Edmond Cary dans les Problèmes théoriques..., p. 13 sqq.; cf. aussi «la Traduction proligère? Sur le statut des textes qu'on traduit», p. 106 sq.

64. «Pour une philosophie de la traduction», p. 9 sq. 
laquelle m'avait prémuni ma formation philosophique et, en particulier, ma fréquentation de la tradition philosophique allemande qui va des néo-kantiens à Jürgen Habermas ${ }^{65}$ - je n'y ai sans doute pas tout à fait échappé et ce n'est que rétrospectivement que j'en ai fait explicitement la critique ${ }^{66}$.

Surtout, il est bien clair que c'est la linguistique qui fournissait l'essentiel des items terminologiques permettant d'étiqueter et de conceptualiser les réalités évidemment langagières avec lesquelles opère la traduction. En outre, la linguistique apportait une méthodologie dont la "scientificité»- au sens que peut prendre, en deçà des naïvetés positivistes, ce concept dans la logique d'une épistémologie des sciences humaines - permettait de constituer l'organon de la traductologie. Le discours sur la traduction accédait ainsi à un statut théorique qui le mettait en mesure de s'affranchir des séductions «littéraires» du je-ne-sais-quoi faisant de la traduction un art, qui échapperait par nature à toute rationalité, et de dépasser les affects d'un practicisme récusant, parallèlement, tout effort de systématisation réflexive ${ }^{67}$.

Ainsi prenait-on congé définitivement (enfin!) de la mythologie essentialiste ou «platonisante» immanente à l'idée ancienne qu'il y a un génie des langues, propre à chacune d'elles cette idée pouvant aller à prouver que la traduction est impossible, alors qu'on voit tout de suite qu'elle était faite pour séduire ceux qui parlaient de traduction. En sorte qu'on retomberait dans la

65. Voir mon étude «le Programme épistémologique de Jürgen Habermas», préfaçant la traduction de J. Habermas, Connaissance et intérêt (Paris, Gallimard, Bibliothèque de Philosophie, 1976; rééd.: coll. «Tel», $\left.\mathrm{n}^{\circ} 38\right)$, p. 7 sqq.

66. «Linguistique appliquée et enseignement de la langue» II, Revue d'Allemagne, t. VII, $\mathrm{n}^{\circ} 4$ (octobre-décembre 1975), p. 525 sqq.

67. Voir notamment «la Traduction proligère? Sur le statut des textes qu'on traduit», p. 103. 
problématique de l'objection préjudicielle que j'ai critiquée (plus haut et dans le troisième chapitre des Théorèmes...).

Il reste que, au-delà de cette première et principale critique adressée aux travaux de Mounin, il en est au moins deux autres qui mettent en cause ce que j'ai appelé son linguisticisme: non seulement Mounin veut à toute force démontrer que les problèmes de la traduction constituent un chapitre de la linguistique (cf. supra), qu'ils définissent une sous-discipline de cette dernière, mais encore il entend utiliser la traduction comme un dispositif vérifiant le postulat méthodologique fondateur de la linguistique structurale et établissant que les langues sont bien des systèmes. À l'instar du bilinguisme, la traduction serait «un moyen particulier de vérifier l'existence et le jeu des structures dans les langues $\left.{ }^{68}\right)$. Il est clair que ce sont là deux questions pour la linguistique, éventuellement, mais pas pour la traduction - et encore moins pour les traducteurs! C'était l'une des raisons qui m'avaient amené à voir dans les Problèmes théoriques de la traduction un Cours de linguistique générale (cf. supra).

\section{Un livre fondateur, quand même}

Plus généralement, ce livre est aussi une Thèse (de doctorat), tenant le discours académique d'un exposé de thèses critiquées l'une après l'autre pour établir le point de vue de l'auteur et, en quelque sorte, pour le «construire» au terme d'un tel parcours argumentatif - d'un tel «périple», pour citer Mounin lui-même. Il s'ensuit qu'il y a là une discursivité qui lui est propre et dont, pour conclure, je voudrais évoquer quelques caractéristiques.

D'abord, cette facture académique qui donne au travail de Mounin l'aspect d'un cours de linguistique fait que c'est souvent du discours didactique que relèvent ses analyses, autant et plus que du

68. Les Problèmes théoriques de la traduction, p. 6. 
«discours scientifique» (stricto sensu) ${ }^{69}$. Cette remarque est encore une critique à l'œuvre de Mounin, mais seulement en partie, dans la mesure où c'est aussi un aspect par lequel elle se montre tout indiquée pour la formation intellectuelle des futurs traducteurs et des apprentis linguistes qui s'intéressent à la traduction.

En outre, la démarche argumentative elle-même fait de Mounin l'avocat d'une cause, celle de la linguistique, et du même coup un polémiste critiquant les thèses de ceux qui dès lors apparaissent comme ses adversaires. Certes, c'est un peu la règle de l'institution académique. Mais, plus encore, il semble que la polémique soit une dimension essentielle aux débats touchant la traduction $^{70}$.

Cela dit, le point que je veux surtout souligner ici, c'est que le mode démonstratif de l'argumentation fait que le discours «traductologique» de Mounin est à certains égards plus proche de la discursivité d'une dissertation philosophique, voire d'une disputatio théologique, que de ce qu'on se représente que peut être un discours de recherche tel qu'on serait en droit de l'attendre d'un travail relevant de la «science» linguistique. Là encore, on pourrait voir dans cette caractérisation une critique; mais ce serait plutôt le contraire, pour (au moins) deux raisons.

D'une part, très généralement, il $\mathrm{y}$ a là un élément d'illustration d'une thèse que je défends en matière d'épistémologie des sciences humaines. Ce n'est en effet que par une analogie abusive que d'aucuns s'imaginent pouvoir déceler l'équivalent pour les sciences humaines de la "coupure épistémologique» qui a institué les sciences exactes comme telles: méconnaître cette vérité relève

69. Voir, sur l'opposition de ces deux modes de discours, «le Discours scientifique», Revue d'ethnopsychologie, t. XXVI, $\mathrm{n}^{\circ}$ 2-3 (septembre 1971), pp. 153-191.

70. «La Traduction proligère? Sur le statut des textes qu'on traduit», p. 103 sqq. 
d'un positivisme dont il convient de faire la critique, ainsi qu'il a été indiqué au début de notre contribution; et ce, même s'il est bien vrai que quelques secteurs spécifiques et bien délimités des sciences humaines relèvent effectivement des sciences exactes en vertu de la méthodologie qu'ils mettent en œuvre, comme c'est le cas de la psychologie expérimentale par exemple ${ }^{71}$. Autrement, d'une façon générale, il y a lieu de rétablir (ou, plutôt, de maintenir) la continuité existant entre philosophie et sciences humaines, en rapport avec ce que j'ai appelé une «troisième culture», mentionnée en section 1 .

D'autre part, plus spécifiquement, ladite continuité prend tout son sens pour ce qui est de la traductologie, au sein du triptyque linguistique-philosophie-psychologieévoqué plus haut. «La traductologie: de la linguistique à la philosophie» - telle est en tout cas la problématique sous les auspices de laquelle il m'est apparu qu'il y avait lieu de subsumer l'ensemble de mes propres travaux sur la traduction ${ }^{72}$. Aussi ne puis-je que me réjouir de ce que Mounin a pressenti cette approche, en dépit de la pente qui l'inclinait à un certain positivisme linguistique - d'où cet hommage archéologique que je lui rends ici. Pour aller vite, je dirai que la traductologie telle que je me suis attaché à la concevoir n'est pas tant une discipline de savoir qu'une discipline de réflexion ${ }^{73}$. Mais pour que la réflexion traductologique permettant $d$ 'anticiper la solution des problèmes que

71. C'est aussi le cas mutatis mutandis de ce que j'ai annoncé sous le nom de "traductologie inductive (ou scientifique)», cf. «Épistémologie de la traduction», p. 41; et c'est ce qu'illustre, par exemple, Jeanne Dancette, Parcours de la traduction. Étude expérimentale du processus de compréhension (Lille, Presses universitaires de Lille, coll. Étude de la traduction, 1995).

72. C'est le titre que j'ai donné à la thèse d'habilitation à diriger des recherches que j'ai récemment soutenue à l'Université de Paris X-Nanterre, le 21 janvier 1995, sous la direction de Michel Arrivé et sous la présidence de Paul Ricœur.

73. «Épistémologie de la traduction», p. 35 sq. 
vise la «traductologie productive ${ }^{74}$ » puisse être mise en œuvre, il s'agit moins de constituer une discipline systématique prenant pour objet tous les aspects de la traduction que de rassembler les éléments d'une culture traductologique, susceptible de mettre la description linguistique en prise sur une conceptualisation dont le principe est proprement philosophique lors même qu'elle convoque les apports interdisciplinaires qui lui sont nécessaires.

C'est donc sur une ouverture programmatique que les limites imparties à la présente étude commandent de «conclure». Au-delà de cet adieu à Georges Mounin que constituent les pages qu'on vient de lire, c'est en effet dans le cadre d'une prochaine étude (encore!) qu'il conviendra de revenir sur la problématique de l'articulation entre linguistique et traductologie ${ }^{75}$...

Jean-René Ladmiral: Université Paris X-Nanterre, 200 avenue de la République, 92001 Nanterre Cedex, France

\section{RÉSUMÉ: À partir de Georges Mounin: esquisse archéologique} - Georges Mounin est l'un des «pères fondateurs» de la traductologie: c'est donc à partir de cet auteur qu'on pourra entreprendre une archéologie de la discipline - au double sens où c'est à la fois vouloir le dépasser, «en partir», et où c'est aussi "partir» de ses travaux. Certes, il faut récuser la problématique de l'objection préjudicielle, qui nous confronte aux apories de l'«intraduisible», en réinscrivant dans l'histoire les problèmes de la traduction. Encore devra-t-on ne pas se contenter d'une traductologie purement linguistique, voire d'une «traductologie de la langue». Plus généralement, dans la foulée de l'héritage de Mounin, on sera amené à poser le problème de l'articulation entre traductologie et

74. Ibid., p. 41 sqq.

75. C'est, en attendant, un point que j'ai abordé dans ma préface à la réédition des Théorèmes..., p. vii sqq. 
linguistique; on en viendra à s'interroger sur le statut du discours traductologique, sur la dimension philosophique qui est la sienne et sur la place qui lui revient dans le champ épistémologique des sciences humaines.

ABSTRACT: From Georges Mounin and Beyond: an Archeological Draft - Georges Mounin is one of the "founding fathers" of Translation Studies. Therefore, he can be considered the "starting point" of an archeology of the discipline in that there is both the desire to go beyond the author, to "transcend" him, but also a willingness to use his works as a basis. Admittedly, one must challenge the concept of untranslatability, which only leads to the impasse of the "untranslatable," by recasting the problem of translation within the context of history. One should not be content with a purely linguistic approach to Translation Studies, or a "translation approach to the study of language." In more general terms, in keeping with the Mounin tradition, the author raises the issue regarding the relation between Translation Studies and Linguistics. He examines the status of translation discourse, its philosophical dimension and its rightful place within the epistemological confines of the Humanities. (Trans. by Paul Bandia) 